\title{
Palmprint Biometric System using Line based Feature Extraction Methods
}

\author{
Sathish $\mathrm{R}^{1}$, Baskar D ${ }^{2}$, Vinod Kumar D ${ }^{3}$ \\ sathishlec25@gmail.com, basindia.kd@gmail.com, vino.kd@gmail.com \\ ${ }^{1}$ Research Scholar - Electronics \& Communication Engineering, ${ }^{3}$ Professor \& Head - Biomedical \\ Engineering, Vinayaka Mission's Kirupananda Variyar Engineering College, Vinayaka Mission's Research \\ Foundation (Deemed to be University), Salem, Tamil Nadu, India. \\ ${ }^{2}$ Assistant Professor - Electrical \& Electronics Engineering, Annai Teresa College of Engineering, \\ Viluppuram, Tamil Nadu, India.
}

\begin{abstract}
Biometrics is the study of estimating human qualities to confirm or recognize the personality of a person. Palmprint is one of the human physiological attributes acquiring consideration among analysts as the mean of security. The Chinese Academy of Sciences Institute of Automation (CASIA) database is used for investigations. Lines or boundaries carry vital information for object recognition. Principal lines, Wrinkles and Ridges are categorized as line features. Competent Line-based feature extraction methods used for various object recognition are selected and discussed. The palmprint line features are extracted using Prewitt Edge Detector, Sobel operator, Canny Edge Detector, Kirsch Operator and Multiscale Edge Detector. In which Kirsch Operator performs good and achieves $94.95 \%$ accuracy for $1 \%$ of FAR and $94.85 \%$ accuracy for $2 \%$ of FAR.
\end{abstract}

Keywords: Biometrics, Palmprint, Authentication, Line features Object Recognition

\section{Introduction}

Biometrics is obtained from the Greek words "Bio" signifes life and "Metrics" signifies to measure. Biometrics is the study of estimating human qualities to confirm or recognize the personality of a person. It is additionally used to recognize people in bunches that are under reconnaissance. Biometrics are mainly classified as physiological and behavioral characteristics.

\section{Palmprint}

Palmprint is one of the human physiological attributes. It is special in light of the fact that each palmprint is unique in relation to other people. It is demonstrated that palmprint contains rich hereditarily disconnected highlights for ordering indistinguishable twins. Palmprint is lasting or indivisible from the individual contrasted with recognizable proof things. Palmprint is not difficult to gather and steady since it doesn't change essentially after some time [1,2,3,4,5]. It can be used to compare digitally with other individuals. Due to its size and features rich it is hard to imitate. It consists of various features such as geometry, point, line, texture, and statistical.

\section{Line-Based Operators}

Principal lines, wrinkles and ridges are the three line features of palmprint. Heart line, life line and head line are the three major types of principal lines. The coarse lines are called as wrinkles and the fine lines are called as ridges. The palmprint line features are shown in Fig 1. To accurately extract the line features is the main challenge in line based feature extraction. The direction of lines on Palmprint is difficult to predict. 


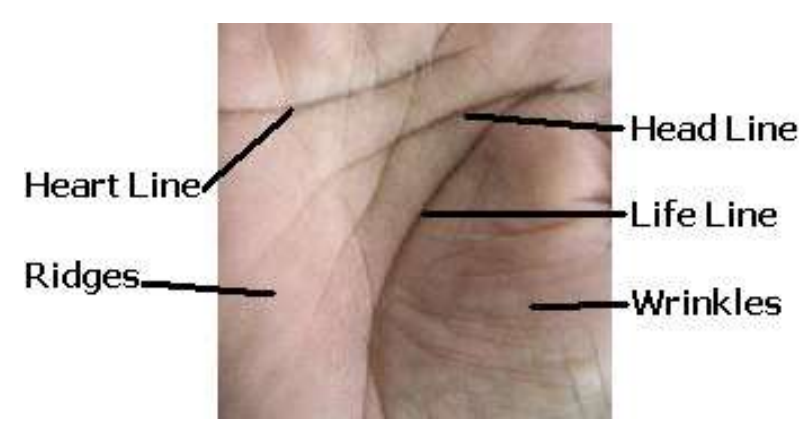

Fig 1. Palmprint Line Features

Lines or boundaries carry vital information for object recognition. There are many line detection methods applied widely for object recognition system. Some of the established line detection methods and certain emerging methods are investigated as feature extraction for palmprint. For remote sensing application Canny edge detector is used for feature extraction the result was robust [8]. Prewitt edge detector is used as a edge detection algorithm in mammographic images. Parametes such as tumor location, brest boundary and pectoral region are viewed clearly. This was used specifically for enhancing the tumor area in mammographic images [9]. Sobel operator is used in applications involving observation framework and clinical determination under low illumination or lack of visible light or medical requirements, thermal imaging [10]. The adaptation and optimization of Sobel and Canny edge detector algorithms are used in various applications such as augmented reality, computer vision \& mobile phone videos processing softwares.

The proposed solution was robust and can be efficiently used in power device which use very low power [11]. Sobel Edge Detection was used to detect vehicle number plates [12]. Various sourrses of noise currupt the real images. Multi Scale edge Detector is used to preserve the quality of the image comparing to linear filters [13]. Multiscale Edge Detection is used in medical ultrasound signals [14].

The Kirsch Edge Detector and Prewitt edge Detector also showed good results in mammographic images [15]. These operators are not investigated for feature rich object like palmprint However these entire operators have demonstrated high efficiency for various computer vision and biomedical applications.

\section{Line Detection for Palmprint}

Palmprint line features can be extracted using Prewitt Edge Detector, Sobel operator, Canny Edge Detector, Kirsch Operator and Multiscale Edge Detector.

\subsection{Prewitt Edge Detector}

Edge detection is found out by differentiating the changes in image intensity. It is important to implement averaging within the edge detection process. The Prewitt edge detection method consist of two templates, $M x \& M y$, over the three columns. The length of the vector is $M$ and $\theta$ is the angle of the vector.

The rate of change of brightness is illustrated in Fig 2. The appropriate quadrant for the edge direction is determined using the signs of $M x$ and $M y$.

$$
M=\sqrt{M x(x, y)^{2}+M y(x, y)^{2}}
$$




$$
\theta(x, y)=\tan ^{-1}\left(\frac{M y(x, y)}{M x(x, y)}\right)
$$

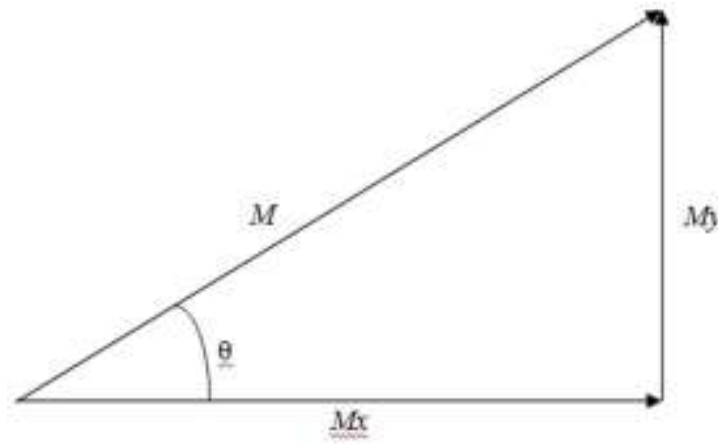

Fig 2. Edge Detection in Vectorial Format

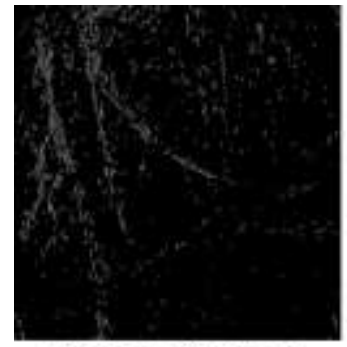

Person 1 Sample 1

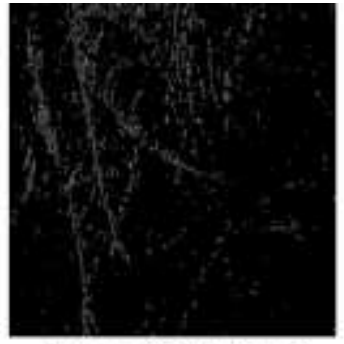

Person 1 Sample 2

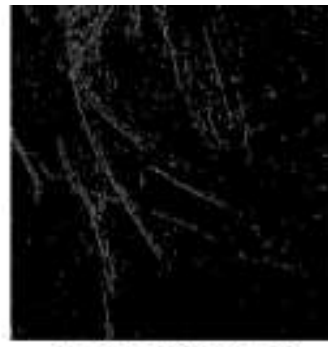

Person 2 Sample 1

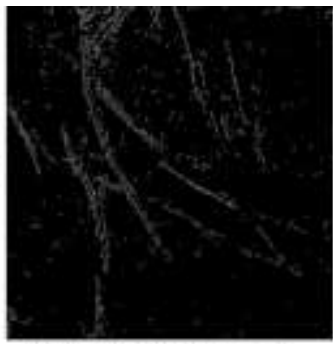

Person 2 Sample 2

Fig 3. Results for Prewitt Edge Detector

Table 1. FAR, FRR and Accuracy Table for Prewitt Edge Detector

\begin{tabular}{|c|c|c|c|}
\hline Threshold & FAR & FRR & Accuracy \\
\hline 0.25 & 0.29 & 0.22 & 74.49 \\
\hline 0.5 & 0.25 & 0.20 & 77.33 \\
\hline 0.75 & 0.22 & 0.19 & 79.44 \\
\hline 1 & 0.18 & 0.18 & $\mathbf{8 1 . 9 9}$ \\
\hline 1.25 & 0.15 & 0.23 & 80.99 \\
\hline 1.5 & 0.11 & 0.26 & 81.47 \\
\hline 1.75 & 0.08 & 0.29 & 81.5 \\
\hline 2 & 0.075 & 0.32 & 80.25 \\
\hline 2.25 & 0.068 & 0.35 & 79.1 \\
\hline 2.5 & 0.057 & 0.39 & 77.65 \\
\hline 2.75 & 0.042 & 0.41 & 77.4 \\
\hline 3 & 0.037 & 0.42 & 77.15 \\
\hline 3.25 & 0.022 & 0.47 & 75.4 \\
\hline 3.5 & 0.015 & 0.48 & 75.25 \\
\hline 3.75 & 0.006 & 0.51 & 74.2 \\
\hline
\end{tabular}




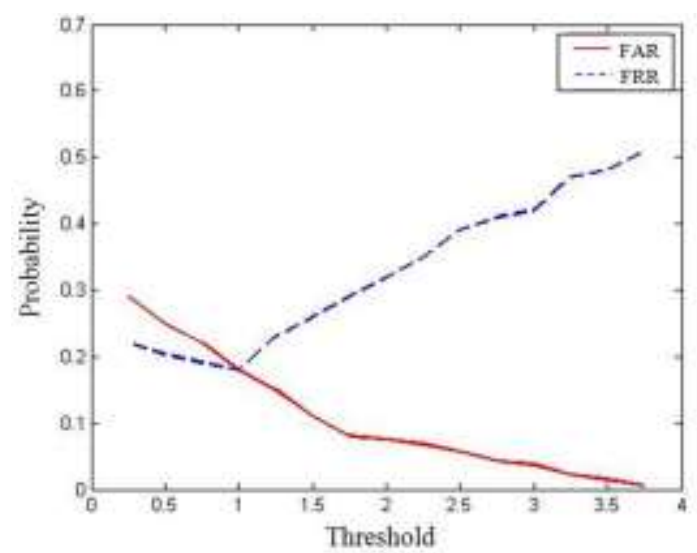

(a) Threshold Vs FAR \& FRR

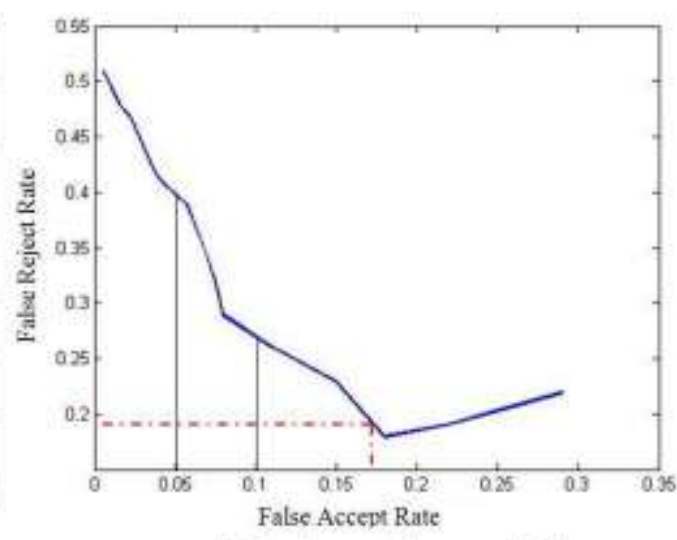

(b) Receiver operating curve (ROC)

Fig 4. Accuracy plot for Prewitt edge detector

Prewitt Edge Detector results on palmprint images are given in Fig 3. From Table 1 it is observed the accuracy at approximate Equal Error rate is $81.995 \%$ and corresponding FAR and FRR values are $18 \%$ and $18.01 \%$ respectively. Prewitt Edge Detector achieves $77.65 \%$ accuracy for 5\% of FAR and $81.5 \%$ accuracy for $10 \%$ of FAR. In Fig 4 Variations for FAR and FRR Vs Threshold and Receiver Operating Curve for Prewitt Edge Detector have been plotted respectively.

\subsection{Sobel Operator}

The sobel operator is popular because of its overall performance over further edge detection operators. Conventional $(3 \times 3)$ Sobel operators are used to detect horizontal and vertical lines.

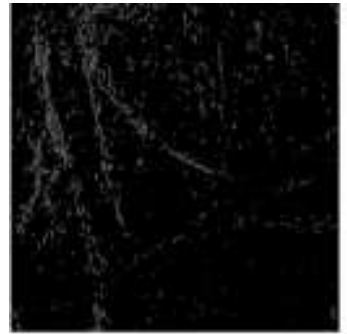

Person 1 Sample 1

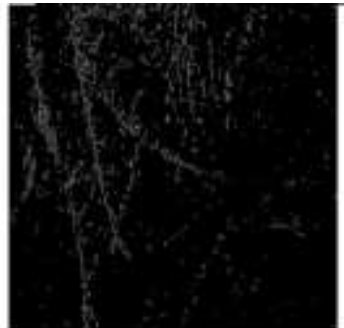

Person 1 Sample 2

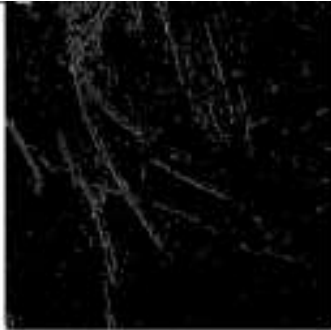

Person 2 Sample 1

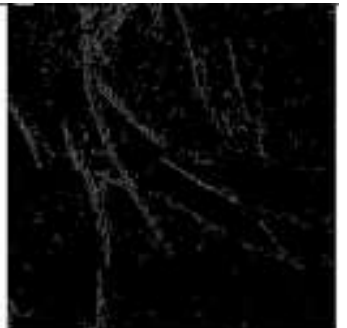

Person 2 Sample 2

Fig 5. Results for Sobel Operator

The results for Sobel Operator on Palmprint images are shown in Fig 5. From Table 2 it is observed the accuracy at approximate Equal Error rate is $84.8 \%$ and corresponding FAR and FRR values are $15.2 \%$ and $15.2 \%$ respectively. It also achieves $79.01 \%$ accuracy for $5 \%$ of FAR and $84.385 \%$ accuracy for $10 \%$ of FAR. In Fig 6 Variations for FAR and FRR Vs Threshold and Receiver Operating Curve for Sobel Operator have been plotted respectively. 
Table 2. FAR, FRR and Accuracy table for Sobel Operator

\begin{tabular}{|c|c|c|c|}
\hline Threshold & FAR & FRR & Accuracy \\
\hline 0.01 & 0.20 & 0.16 & 81.55 \\
\hline 0.02 & 0.19 & 0.15 & 82.6 \\
\hline 0.03 & 0.18 & 0.15 & 83.1 \\
\hline 0.04 & 0.17 & 0.14 & 84.25 \\
\hline 0.055 & 0.15 & 0.15 & $\mathbf{8 4 . 8}$ \\
\hline 0.06 & 0.13 & 0.18 & 84.35 \\
\hline 0.075 & 0.11 & 0.19 & 84.54 \\
\hline 0.08 & 0.09 & 0.22 & 84.38 \\
\hline 0.09 & 0.07 & 0.28 & 82.20 \\
\hline 0.1 & 0.05 & 0.37 & 79.01 \\
\hline 0.25 & 0.03 & 0.39 & 78.51 \\
\hline 0.55 & 0.02 & 0.41 & 78.08 \\
\hline 0.75 & 0.011 & 0.42 & 78.38 \\
\hline 1.1 & 0.01 & 0.45 & 77.27 \\
\hline 1.5 & 0.004 & 0.48 & 75.68 \\
\hline
\end{tabular}

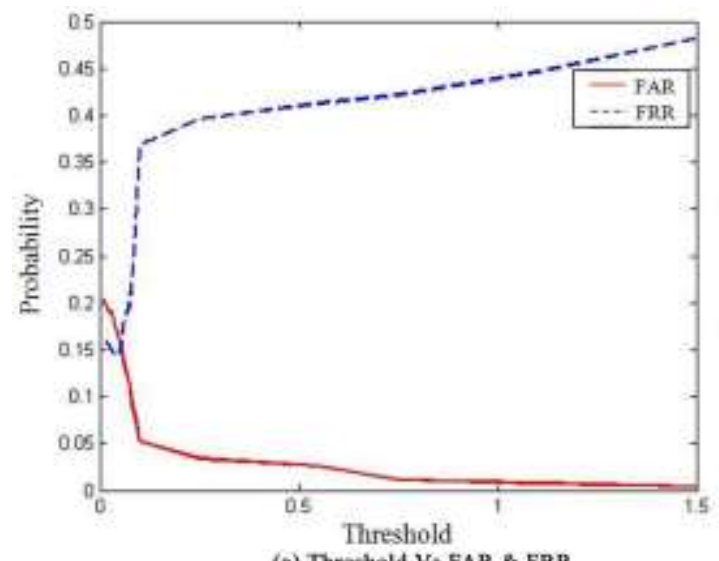

(a) Threshold Vs FAR \& FRR

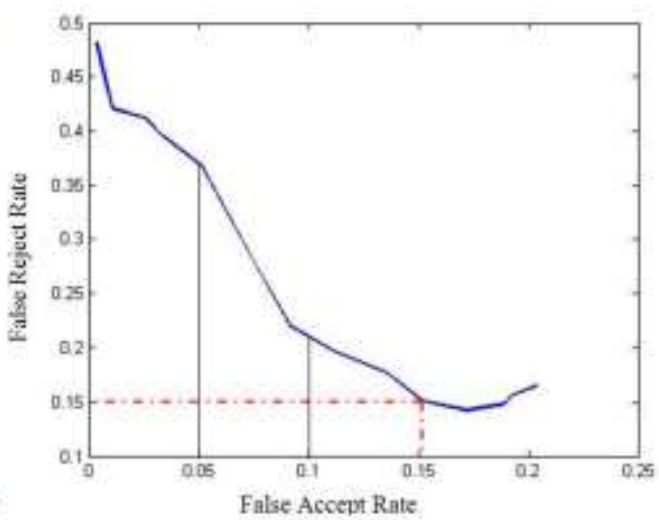

(b) Receiver operating curve (ROC)

Fig 6. Accuracy plot for Sobel edge detector

\subsection{Canny Edge Detector}

The main objectives of Canny edge detector are Optimal detection, good localisation and true edge position. The signal to noise ratio is over come by Non linier suppression. It concerns location of a single edge point in response to a change in brightness. To detect the wide range of images multi stage algorithm is used. The maximum value of the edge is detected using gradient intensity matrix. 


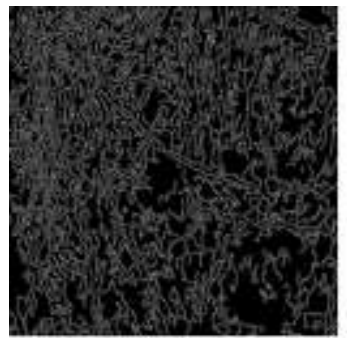

Person 1 Sample 1

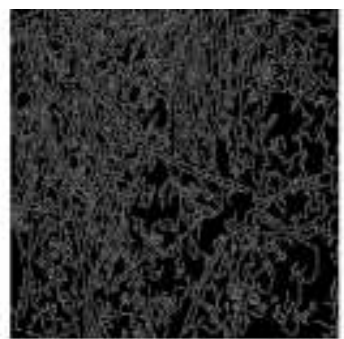

Person 1 Sample 2

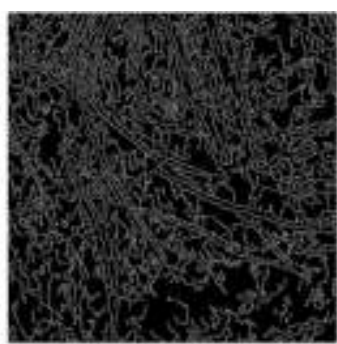

Person 2 Sample 1

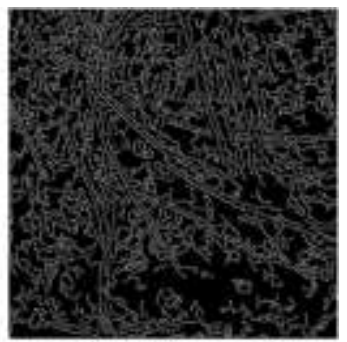

Person Sample 2

Fig 7. Results using Canny Edge Detector

Table 3: FAR, FRR and Accuracy Table for Canny Edge Detector

\begin{tabular}{|c|c|c|c|}
\hline Threshold & FAR & FRR & Accuracy \\
\hline 0.25 & 0.14 & 0.11 & 87.51 \\
\hline 0.5 & 0.14 & 0.11 & 87.12 \\
\hline 0.75 & 0.12 & 0.12 & $\mathbf{8 7 . 8 4}$ \\
\hline 1 & 0.11 & 0.14 & 87.63 \\
\hline 1.25 & 0.10 & 0.15 & 87.72 \\
\hline 1.5 & 0.10 & 0.15 & 87.51 \\
\hline 1.75 & 0.09 & 0.16 & 87.69 \\
\hline 2 & 0.08 & 0.17 & 87.70 \\
\hline 2.25 & 0.07 & 0.18 & 87.55 \\
\hline 2.5 & 0.06 & 0.19 & 87.62 \\
\hline 2.75 & 0.05 & 0.20 & 87.78 \\
\hline 3 & 0.04 & 0.21 & 87.7 \\
\hline 3.25 & 0.03 & 0.22 & 87.72 \\
\hline 3.5 & 0.01 & 0.24 & 87.39 \\
\hline 3.75 & 0.01 & 0.25 & 87.69 \\
\hline
\end{tabular}

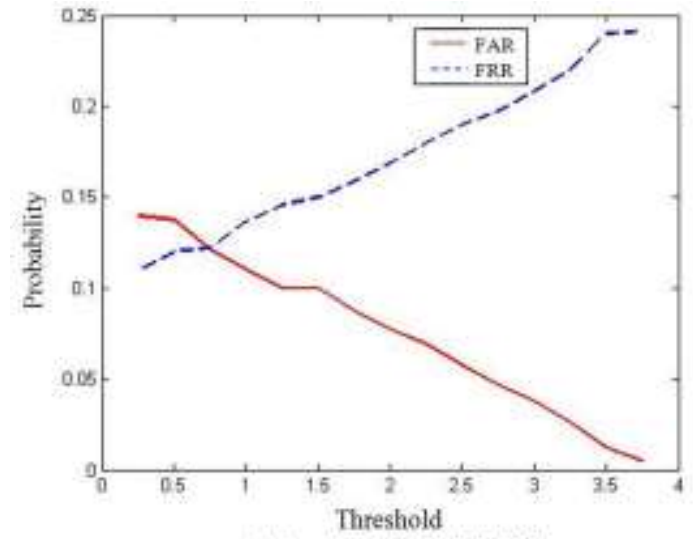

(a) Threshold Vs FAR \& FRR

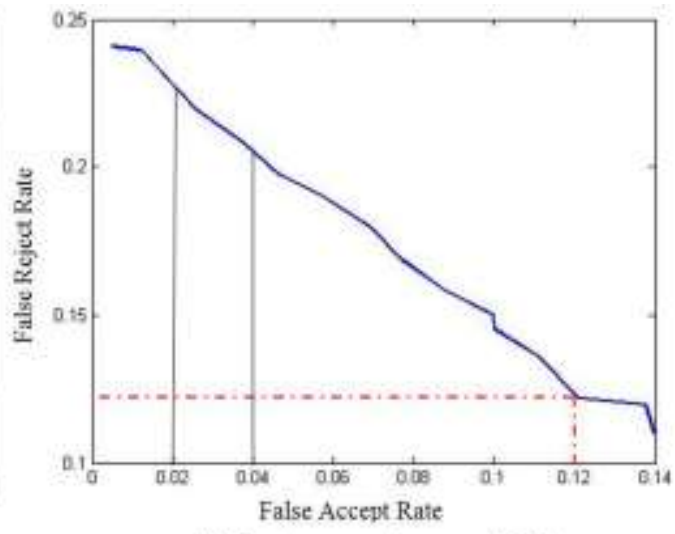

(b) Receiver operating curve (ROC)

Fig 8: Accuracy plot for Canny edge detector 
The results for Canny Edge Detector on palmprint images are shown in Fig 7. From the Table 3 it is observed the accuracy at approximate Equal Error rate is $87.84 \%$ and corresponding FAR and FRR values are $12.11 \%$ and $12.21 \%$ respectively. It also achieves $87.715 \%$ accuracy for $2 \%$ of FAR and $87.785 \%$ accuracy for $10 \%$ of FAR. In Fig 8. Variations for FAR and FRR Vs Threshold and Receiver Operating Curve for Canny Edge Detector have been plotted respectively.

\subsection{Kirsch Operator}

The Kirsch operator is an edge detection method that can find the maximum edge strength in eight predetermined directions or say using eight compass filters. The eight major compass orientations are respectively, N, NW, W, SW, S, SE, E, and NE. These filters are applied to the image with the maximum being retained for the final image.

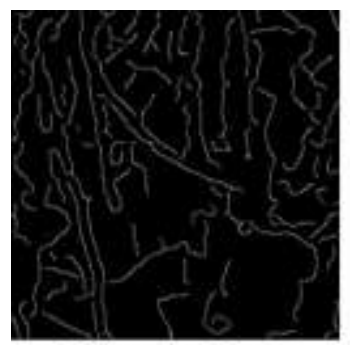

Person 1 Sample 1

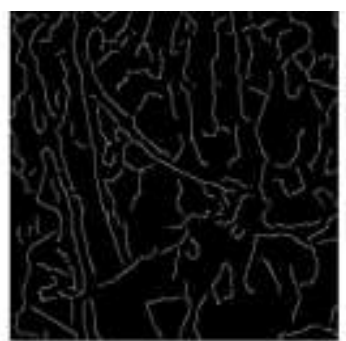

Person 1 Sample 2

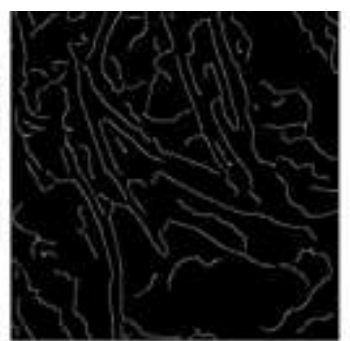

Person 2 Sample 1

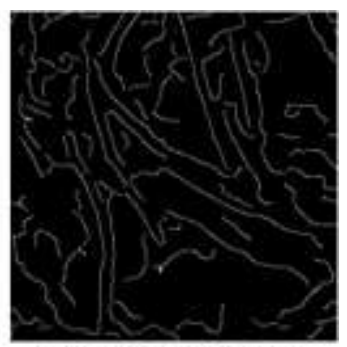

Person 2 Sample 2

Fig 9. Results using Kirsch Operator

Table 4:FAR, FRR and Accuracy Plot for Kirsch Operator

\begin{tabular}{|c|c|c|c|}
\hline Threshold & FAR & FRR & Accuracy \\
\hline 1.3 & 0.085 & 0.021 & 94.69 \\
\hline 1.7 & 0.079 & 0.028 & 94.64 \\
\hline 2.1 & 0.073 & 0.031 & 94.82 \\
\hline 2.5 & 0.07 & 0.037 & 94.7 \\
\hline 2.9 & 0.06 & 0.041 & 94.89 \\
\hline 3.3 & 0.05 & 0.049 & $\mathbf{9 4 . 9 5}$ \\
\hline 3.7 & 0.05 & 0.052 & 94.85 \\
\hline 4.1 & 0.05 & 0.057 & 94.74 \\
\hline 4.5 & 0.04 & 0.061 & 94.85 \\
\hline 4.9 & 0.037 & 0.065 & 94.89 \\
\hline 5.3 & 0.03 & 0.073 & 94.74 \\
\hline 5.7 & 0.028 & 0.078 & 94.69 \\
\hline 6.1 & 0.02 & 0.084 & 94.85 \\
\hline 6.5 & 0.01 & 0.089 & 94.95 \\
\hline 6.9 & 0.01 & 0.099 & 94.75 \\
\hline
\end{tabular}




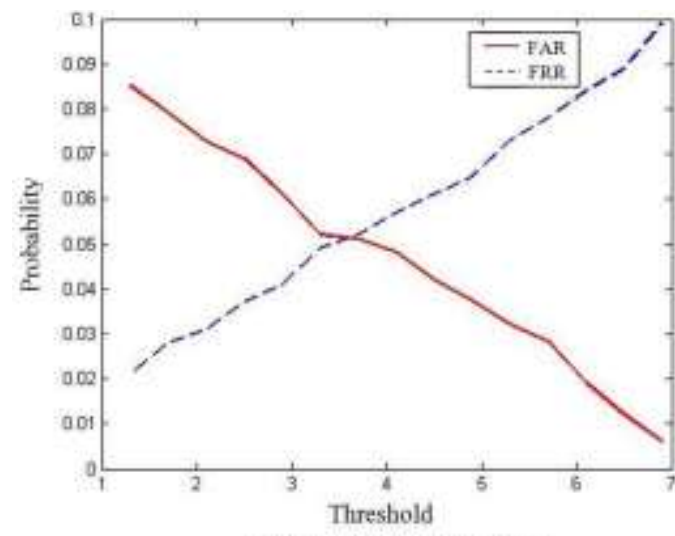

(a) Thresbold Vs FAR \& FRR

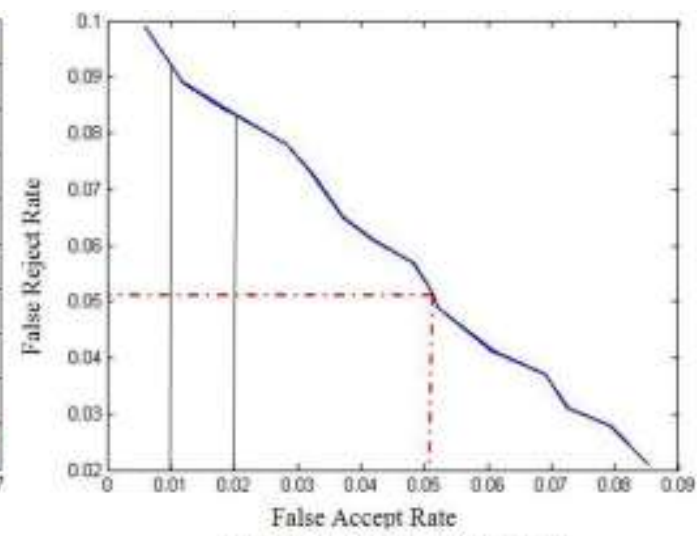

(b) Receiver operating curve (ROC)

Fig 10. Accuracy plot for Kirsch edge detector

The results for Krisch Operator on Palmprint images are shown in Fig 9. From the Table 4 it is observed the accuracy at approximate Equal Error rate is $94.945 \%$ and corresponding FAR and FRR values are 5.21\% and 4.9\% respectively. It also achieves $94.945 \%$ accuracy for $1 \%$ of FAR and $94.845 \%$ accuracy for $2 \%$ of FAR. In Fig 10. Variations for FAR and FRR Vs Threshold and Receiver Operating Curve for Kirsch Operator have been plotted respectively.

\subsection{Multiscale Edge Detector}

In Multiscale Edge Detection method the aim is to simultaneously extract edges of all lengths, in both natural and noisy images. In order to minimize the amount of data edge detection is used. It is used to find the boundaries inside an image. It works by detecting disjointedness in brightness. The brightness of the image varies sharply are called the edges. Convolution techniques are used to to process high resulation images.

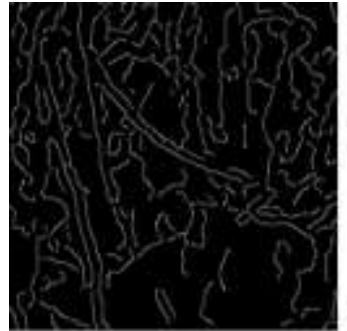

Person 1 Sample 1

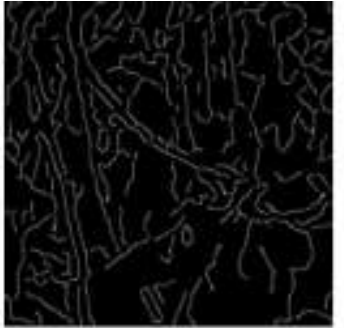

Person 1 Sample 2

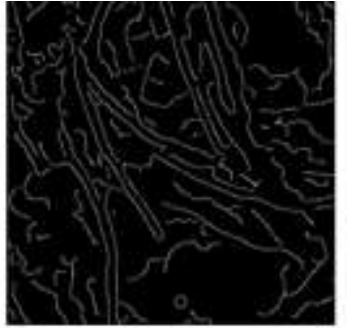

Person 2 Sample 1

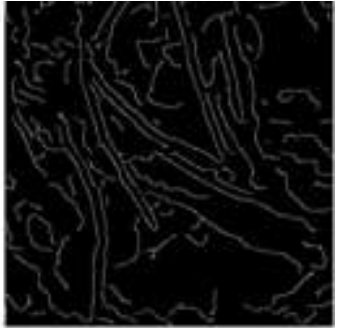

Person 2 Sample 2

Fig 11: Results using Multiscale Edge Detector

The results for Multiscale Edge Detector on Palmprint images are shown in Fig 11. From Table 5 it is observed the accuracy at approximate Equal Error rate is $93.8 \%$ and corresponding FAR and FRR values are $6.4 \%$ and $6 \%$ respectively. It also achieves $93.28 \%$ accuracy for $1 \%$ of FAR and $94.045 \%$ accuracy for $2 \%$ of FAR. In Fig 12 Variations for FAR and FRR Vs Threshold and Receiver Operating Curve for Multiscale Edge Detector have been plotted respectively. 
Table 5: FAR, FRR and Accuracy Table for Multiscale Edge Detector

\begin{tabular}{|c|c|c|c|}
\hline Threshold & FAR & FRR & Accuracy \\
\hline 0.05 & 0.095 & 0.029 & 93.8 \\
\hline 0.08 & 0.081 & 0.0309 & 94.405 \\
\hline 0.1 & 0.078 & 0.041 & 94.05 \\
\hline 0.13 & 0.075 & 0.048 & 93.85 \\
\hline 0.16 & 0.07 & 0.052 & 93.9 \\
\hline 0.17 & 0.064 & 0.06 & $\mathbf{9 3 . 8}$ \\
\hline 0.18 & 0.062 & 0.06 & 93.9 \\
\hline 0.19 & 0.059 & 0.063 & 93.9 \\
\hline 0.21 & 0.046 & 0.072 & 94.1 \\
\hline 0.23 & 0.038 & 0.087 & 93.75 \\
\hline 0.24 & 0.032 & 0.08 & 94.4 \\
\hline 0.26 & 0.021 & 0.093 & 94.3 \\
\hline 0.29 & 0.0201 & 0.099 & 94.045 \\
\hline 0.33 & 0.0124 & 0.122 & 93.28 \\
\hline 0.35 & 0.005 & 0.14 & 92.75 \\
\hline
\end{tabular}

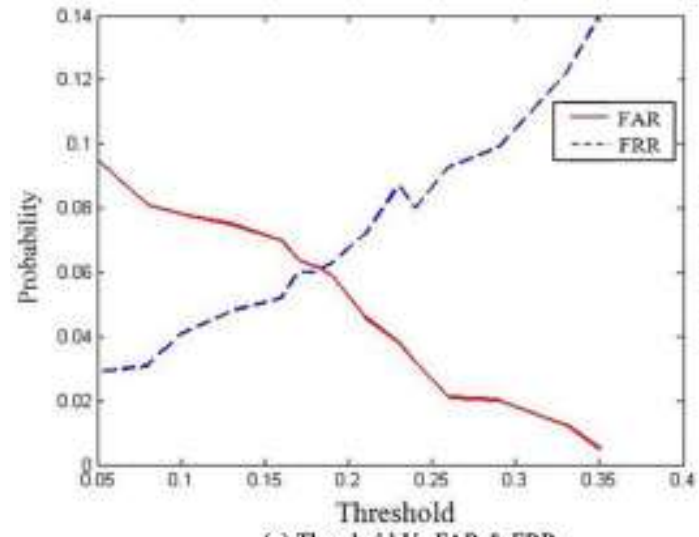

(a) Threshold Vs FAR \& FRR

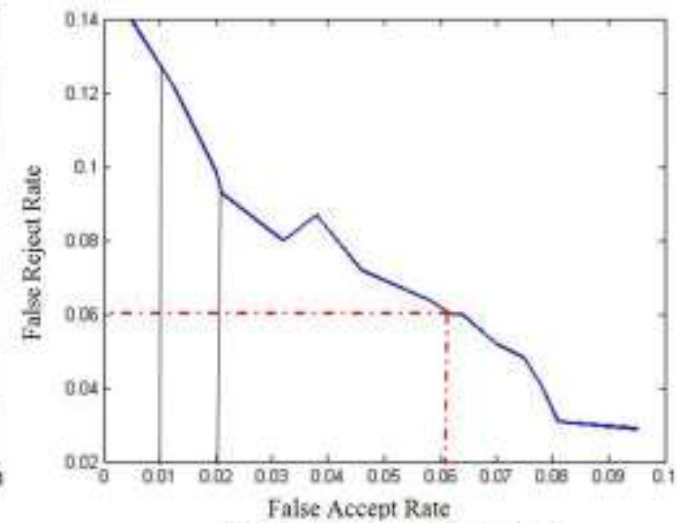

(b) Receiver operating curve (ROC)

Fig 12: Accuracy plot for Multi-Scale edge detector

\section{Result \& Discussion}

The line-based feature extraction method is applied on Palmprint extracted in the preprocessing stage. The graphs and tables corresponding to various line feature extraction methods such as Prewitt Edge Detector, Sobel Operator, Canny Edge Detector, Kirsch Operator and Multiscale Edge Detector are discussed. Table 6. presents the comparison of FAR, FRR and Accuracy of various Line based methods analysed. Based on the analysis Kirsch Operator shows better results than other line based methods. It achieves $94.945 \%$ accuracy for $1 \%$ of FAR and $94.845 \%$ accuracy for $2 \%$ of FAR. 
Table 6: Comparison of FAR, FRR and Accuracy of various Line Based Methods

\begin{tabular}{|l|c|c|c|}
\hline Method Name & $\begin{array}{c}\text { Accuracy } \\
\text { FAR = FRR }\end{array}$ & $\begin{array}{c}\text { Accuracy for } \\
\text { (FAR \%) }\end{array}$ & $\begin{array}{c}\text { Accuracy for } \\
\text { (FAR \%) }\end{array}$ \\
\hline PREWITT & 81.995 & $77.65(5 \%)$ & $81.5(10 \%)$ \\
\hline SOBEL & 84.8 & $79.01(5 \%)$ & $84.385(10 \%)$ \\
\hline CANNY & 87.84 & $87.715(2 \%)$ & $87.785(10 \%)$ \\
\hline MUTISCALE & 93.8 & $93.28(1 \%)$ & $94.045(2 \%)$ \\
\hline KIRSCH & $\mathbf{9 4 . 9 4 5}$ & $\mathbf{9 4 . 9 4 5 ( 1 \% )}$ & $\mathbf{9 4 . 8 4 5 ( 2 \% )}$ \\
\hline
\end{tabular}

\section{Conclusion}

Individual verification utilizing palmprint is acquiring fame as a result of palmprint being a component rich and carefully designed biometric. The line based methods are scrutinized with MATLAB programming. The increase in FAR prompts to less security or legitimate verification leads to accept any person as genuine. Various performance measures such as FAR, FRR, ERR and Accuracy used to evaluate the performance of a biometric systems are discussed. Efficient line-based feature extraction methods used for various object recognition are selected and those methods are implemented on palmprint and their efficiency is analyzed. Based on the analysis Kirsch Operator shows better results than other line based methods. It achieves $94.945 \%$ accuracy for $1 \%$ of FAR and $94.845 \%$ accuracy for $2 \%$ of FAR.

\section{References}

[1] Muthukrishnan, A.; Charles Rajesh Kumar, J.; Vinod Kumar, D.; Kanagaraj, M. Internet of image things-discrete wavelet transform and Gabor wavelet transform based image enhancement resolution technique for IoT satellite applications. Cogn. Syst. Res. 2019, 57, 46-53.

[2] D. Vinod Kumar, A. Nagappan. "Performance Analysis of Security and Accuracy on Palmprint Based Biometric Authentication System," International Journal of Innovative Research in Computer and Communication-Engineering, Vol. 3, Issue 7, July 2015, pp 6697-6704.

[3] Charles Rajesh Kumar.J , Vinod Kumar.D, Baskar.D, Mary Arunsi.B, Jenova.R, ,M.A.Majid." VLSI design and implementation of High-performance Binary-weighted convolutional artificial neural networks for embedded vision based Internet of Things (IoT)".16th International Learning \& Technology Conference (L\&T), Procedia Computer science (Elsevier), Jeddah,2018.

[4] D. Vinod Kumar, A. Nagappan. "Study and comparison of various point based feature extraction methods in palmprint authentication system," International Journal of Computational Engineering Research (IJCER), Vol. 2, Issue 8, December 2012, pp 82-89.

[5] D. Vinod Kumar, A. Nagappan, Jyoti Malik "Phase Congruency Corner Detection as Biometric Feature," IRACST - International Journal of Computer Science and Information Technology \& Security (IJCSITS), Vol. 2, Issue 2, February 2012, pp 179-185.

[6] Kong A. W. K., Zhang D. and Lu G., "A study of identical twins' palmprints for personal verification”, Pattern Recognition, Vol. 39, Issue 11, Pages 2149-2156, November 2006.

[7] Baskar. D, Selvam. P, "Machine Learning framework for Power System Fault Detection and Classification," International Journal of Scientific \& Technology Research, vol. 9, no. 2, pp. 2002-2008, Feb. 2020.

[8] Mohamed Ali and David Clausi, "Using The Canny Edge Detector for Feature Extraction and Enhancement of Remote Sensing Images”, 7803-7031-7/01IEEE, Pages 2298-2300, January 2001.

[9] Chunsun Zhang, Emmanuel Baltsavias and Armin Gruen, "Knowledge-Based Image Analysis for 
3D Road Reconstruction”, Asian Journal of Geoinfomatics, Vol.1, No. 4, Pages 3-14, June 2001.

[10] Ching Wei Wang, "Real Time Sobel Square Edge Detector for Night Vision Analysis", ICIAR 2006, LNCS 4141, Springer-Verlag Berlin Heidelberg Pages 404-413, May 2006.

[11] Marcelo G. Roque, Rafael M. Musmanno, Anselmo Montenegro and Esteban W. G. Clua, "Adapting the Sobel Edge Detector and Canny Edge Extractor for iPhone 3GS architecture", IWSSIP 2010 - 17th International Conference on Systems, Signals and Image Processing, Pages 486-489, Dec 2010.

[12] Suri P.K., Ekta Walia and Amit Verma, "Vehicle Number Plate Detection using Sobel Edge Detection Technique", International Journal on Computer Science and Technology (IJCST) Vol. 1, Issue 2, Pages 179-182, December 2010.

[13] Qimei Hu, Xiangjian He and Jun Zhou, "Multi-Scale Edge Detection with Bilateral Filtering in Spiral Architecture", The Pan-Sydney Area Workshop on Visual Information Processing (VIP) 2003, Sydney. Conferences in Research and Practice in Information Technology, Vol. 36, Pages, Nov 2003.

[14] Preben Gråberg Nes, "Fast multi-scale edge-detection in medical ultrasound signals", Computer Vision and Pattern Recognition (cs.CV); Medical Physics (physics.med-ph), Vol. 1, July 2011.

[15] Sumit Chopra, Pankaj Bhambri and Baljit Singh, "Segmentation of the Mammogram Images to find Breast Boundaries", IJCST Vol. 2, Issue 2, Pages 164-167, June 2011. 